\title{
Finite geometric spaces, Steiner systems and cooperative games
}

\author{
Antonio Maturo and Fabrizio Maturo
}

\begin{abstract}
Some relations between finite geometric spaces and cooperative games are considered. The games associated to Steiner systems, in particular projective and affine planes, are considered. The properties of winning and blocking coalitions are investigated.
\end{abstract}

\section{Introduction}

A first study on relations between projective planes and cooperative games is in [38], where the points of the Fano plane are considered to be the players of a cooperative game and the lines are winning coalitions. A formalization of semi-simple cooperative games as a set of players with a set of winning coalitions is in [33]. Some relations between projective planes and cooperative games are introduced in [31]. The research of blocking coalitions gave rise to the geometric theory of blocking sets, studied by various authors (see, e.g., [2], [3], [4], [5], [6], [8], [9], [10], [11], [12], [22], [23], [24], [35], [37]).

In this paper we wish to deepen some aspects of the relationship between semi-simple cooperative games and finite geometric spaces. We introduce the concepts of cooperative games associated to geometric spaces and geometric spaces associated to semi-simple cooperative games. In particular the rank 2 geometries, with particular reference to Steiner systems, are investigated.

Some new results on blocking coalitions are presented.

Key Words: Finite geometries, cooperative games, blocking coalitions, Steiner systems. 2010 Mathematics Subject Classification: Primary 91A12, 05B25; Secondary 51E10, $51 \mathrm{E} 05,20 \mathrm{~N} 20$

Received: September, 2013.

Revised: November, 2013.

Accepted: November, 2013. 


\section{Finite geometric spaces}

Let us recall some fundamental definitions (see, e.g., [7], [14], [16], [19], [36]).

Definition 2.1. A geometry is a pair $G=(\Omega, I)$, with $\Omega$ a non-empty set, called the support and $I$ is a symmetric and reflexive relation on $\Omega$, i.e. such that:

- $\forall(x, y) \in \Omega^{2},(x, y) \in I \Rightarrow(y, x) \in I$;

- $\forall x \in \Omega,(x, x) \in I$.

In the sequel, if $(x, y) \in I$, we say that $x$ and $y$ are incident and we write $x I y$ or $y I x$.

Definition 2.2. Let $G=(\Omega, I)$ be a geometry. $A$ flag of $G$ is a set $\mathcal{F}$ of elements of $\Omega$ that are mutually incident. A flag $\mathcal{F}$ is called maximal if there is no element $x \in \Omega-\mathcal{F}$ such that $\mathcal{F} \cup\{x\}$ is also a flag.

Definition 2.3. We say that a geometry $G=(\Omega, I)$ has rank $\mathrm{k}(k \geq 2)$, we write $G=\left(\Omega_{1}, \Omega_{2}, \ldots, \Omega_{k}, I\right)$, if there exists a finite sequence $\left(\Omega_{1}, \Omega_{2}, \ldots, \Omega_{k}\right)$ of non-empty subsets of $\Omega$ such that:

- $\left\{\Omega_{1}, \Omega_{2}, \ldots, \Omega_{k}\right\}$ is a partition of $\Omega$;

- each maximal flag of $G$ intersects each set $\Omega_{i}$ in exactly one element.

The elements of $\Omega_{i}$ are called elements of type $i$.

Remark 2.1. (Notation with "contains") Let $G=\left(\Omega_{1}, \Omega_{2}, \ldots, \Omega_{k}, I\right)$ be a geometry of rank $k$. In the usual geometric notation, if $x \in \Omega_{i}, y \in \Omega_{j}, i \leq$ $j, x I y$, we say also that " $x$ is contained in $y$ " or " $y$ contains $x$ ".

Remark 2.2. (Notation with "belongs") In an alternative geometric notation, if $x \in \Omega_{i}, y \in \Omega_{j}, i<j, x I y$, we say also that $x$ is belonging to $y$. From this point of view, $y$ can be seen as the set of $x \in \Omega_{i}$ such that $x I y$.

For $k=2$ we have the following definition.

Definition 2.4. A geometry of rank 2 (called also geometric space or incidence structure) is a geometry $G=(\Omega, I)$ where one can partition $\Omega$ into two sets $\mathcal{P}, \mathcal{B}$ such that:

- $\forall x \in \mathcal{P}, \exists y \in \mathcal{B}: x I y$;

- $\forall y \in \mathcal{B}, \exists x \in \mathcal{P}: x I y$;

- $\left((x, y) \in \mathcal{P}^{2} \cap I\right) \vee\left((x, y) \in \mathcal{B}^{2} \cap I\right) \Rightarrow x=y$. 
The elements of $\mathcal{P}$ are called points and the elements of $\mathcal{B}$ blocks. In this case one also writes $G=(\mathcal{P}, \mathcal{B}, I)$.

The blocks are called lines if for any two distinct points $P$ and $Q$ there is exactly one block, called line $P Q$, that is incident with $P$ and $Q$.

In the geometric notation we can identify every block with the set of the points incident with it. If $r$ and $s$ are two blocks we denote with $r \cap s$ the set of the points that are incident with $r$ and $s$. If $r \cap s \neq \emptyset$, i.e. there exists a point $P$ such that $P I r$ and PIs, we say that $r$ intersects $s$.

Definition 2.5. A projective space is a geometric space $G=(\mathcal{P}, \mathcal{B}, I)$ such that (see e.g. [7], [36]):

(PS1) (line axiom). For any two distinct points $P$ and $Q$ there is exactly one line that is incident with $P$ and $Q$, called the line $P Q$;

(PS2) (Veblen-Young axiom) Let $A, B, C, D$ four distinct points such that $A B$ intersects $C D$. Then $A C$ intersects $B D$.

(PS3) Any line is incident with at least three points.

A projective space $G=(\mathcal{P}, \mathcal{B}, I)$ is said to be nondegenerate if:

(PS4) There are three points not incident to the same line.

Definition 2.6. A projective plane (or projective space with dimension 2) is a nondegenerate projective space $G=(\mathcal{P}, \mathcal{B}, I)$ in which the axiom (PS2) is replaced by the stronger axiom:

(PS2S) Two lines intersect in at least a point.

From now on we suppose that the projective spaces we consider are nondegenerate.

Remark 2.3. From the previous axioms it follows that a projective plane has at least 7 points and 7 lines. It is well known that there exists a projective plane with 7 points and 7 lines, called the Fano plane ([7], [19]).

Definition 2.7. If $A, B$ are points of a projective space $G=(\mathcal{P}, \mathcal{B}, I)$, the line $A B$ is said to be the 1-dimensional subspace of $\mathcal{P}$ spanned or generated by the set $\{A, B\}$. If $G=(\mathcal{P}, \mathcal{B}, I)$ is nondegenerate, for any points $A, B, C$ distinct and such that $C$ doesn't belong to the line $A B$, we define plane $A B C$, or 2-dimensional subspace of $\mathcal{P}$ spanned or generated by the set $\{A, B, C\}$, the union of the lines $C X$, with $X \in A B$.

For recurrence we can consider projective spaces and subspaces with greater dimensions. 
Definition 2.8. Let $\mathcal{A}=\left\{A_{1}, A_{2}, \ldots, A_{n}, A_{n+1}\right\}, n \geq 2$, be a set of points of $\mathcal{P}$ such that:

(SP1) The set $\left\{A_{1}, A_{2}, \ldots, A_{n}\right\}$ spans a (n-1)-dimensional subspace $S_{n-1}$ of $\mathcal{P}$

(SP2) $A_{n+1}$ doesn't belong to $S_{n-1}$;

We define $\mathrm{n}$-dimensional subspace of $\mathcal{P}$ spanned or generated by the set $\mathcal{A}$, the union of the lines $A_{n+1} X$, with $X \in S_{n-1}$.

Definition 2.9. Let $G=(\mathcal{P}, \mathcal{B}, I)$ be a nondegenerate projective space. We say that the dimension of $G$ is $n$ if there exists a $n$-dimensional subspace equal to $\mathcal{P}$. In this case the (n-1)-dimensional subspaces are called hyperplanes.

Remark 2.4. In particular:

- a degenerate projective space has dimension 1;

- a projective plane is a projective space with dimension 2 and the hyperplanes are the lines;

- a projective space with dimension greater than 2 has dimension 3 if and only if a line and a plane have at least a point in common.

Definition 2.10. A geometric space is finite if the sets $\mathcal{P}, \mathcal{B}$ are finite.

Let $G=(\mathcal{P}, \mathcal{B}, I)$ be a projective space. We have the following results ([7], [16], [19]):

Proposition 2.1. If a line has exactly $q+1$ points, $q>2$, then all the lines have the some number of points. The number $q$ is said to be the order of $G$.

Proposition 2.2. If $G=(\mathcal{P}, \mathcal{B}, I)$ is a projective space of dimension $d$ and order $q$ then the number of points, equal to the number of hyperplanes, is:

$$
\Theta_{d}(q)=q^{d}+q^{d-1}+\ldots+q+1 .
$$

The number of lines through a fixed point of $G$, equal to the number of points of a hyperplane, is:

$$
\Theta_{d-1}(q)=q^{d-1}+\ldots+q+1 .
$$

Corollary 2.1. If $G=(\mathcal{P}, \mathcal{B}, I)$ is a projective plane then the number of points of $G$, equal to the number of lines, is:

$$
\Theta_{2}(q)=q^{2}+q+1 .
$$


The number of lines through a fixed point of $G$, equal to the number of points of a line, is:

$$
\Theta_{1}(q)=q+1
$$

\section{Cooperative games}

Let us recall some fundamental definitions on cooperative games (see, e.g., [21], [26], [27], [30], [32], [38]).

Let $\mathcal{P}=\left\{P_{1}, P_{2}, \ldots, P_{n}\right\}, n \geq 2$ be a finite set, called the set of players.

Definition 3.1. A function $v: 2^{\mathcal{P}} \rightarrow R$ such that:

(C1) $v(\emptyset)=0$;

(C2) (superadditivity) $\forall A, B \in 2^{\mathcal{P}},(A \cap B)=\emptyset \Rightarrow v(A \cup B) \geq v(A)+v(B)$;

is called the characteristic function on $\mathcal{P}$. The pair $C=(\mathcal{P}, v)$ is called the cooperative game with $n$ players and the subsets of $\mathcal{P}$ are called coalitions.

Remark 3.1. For every $A \in \mathcal{P}$ the number $v(A)$ is interpreted as the total gain (utility) that the players belonging to $A$ can have certainly forming a coalition, independently on the actions of the players not belonging to A. We assume the condition of "side payment", i.e. in every coalition A any player can transfer an amount of his gain to another player belonging to $A$ and so it is important only the total gain of the coalition. The condition (C2) is a consequence of the fact that the total gain obtained with an alliance between two disjoint coalitions is not inferior to the one without cooperation.

We write $v(i)$ to denote $v\left(P_{i}\right)$. By $(\mathrm{C} 2)$ it follows that in a cooperative game $C=(\mathcal{P}, v)$ we have

$$
v(\mathcal{P}) \geq \sum_{i=1}^{n} v(i) .
$$

If

$$
v(\mathcal{P})>\Sigma_{i=1}^{n} v(i)
$$

the game is said to be essential. If, in (5), the equality holds the game is inessential.

It is easy to prove that a cooperative game is inessential if and only if the additive property holds:

$$
\forall A, B \in 2^{\mathcal{P}},(A \cap B)=\emptyset \Rightarrow v(A \cup B)=v(A)+v(B)
$$

and so there are no advantages by any cooperation. 
Definition 3.2. Two cooperative games $C=(\mathcal{P}, v)$ and $C^{\prime}=\left(\mathcal{P}, v^{\prime}\right)$, with the same set of $n$ players, are called strategically equivalent, we write $(\mathcal{P}, v) \approx$ $\left(\mathcal{P}, v^{\prime}\right)$ if there exist $n+1$ real numbers $k>0$ and $c_{1}, c_{2}, . ., c_{n}$ such that:

$$
\forall A \in 2^{\mathcal{P}}, v^{\prime}(A)=k v(A)+\Sigma_{P_{i} \in A} c_{i} .
$$

Remark 3.2. We obtain the game $\left(\mathcal{P}, v^{\prime}\right)$ by the game $(\mathcal{P}, v)$ with an initial payment $c_{r}$ to any player $P_{r}$ and by multiplying the total gain of any coalition by the scale factor $k$. Then we can assume the same strategies to solve $(\mathcal{P}, v)$ or $\left(\mathcal{P}, v^{\prime}\right)$.

Remark 3.3. Let $C=(\mathcal{P}, v)$ a cooperative game. The system with $n+1$ equations and $n+1$ unknowns $k>0$ and $c_{1}, c_{2}, \ldots, c_{n}$ :

$$
v^{\prime}(\mathcal{P})=k v(\mathcal{P})+\Sigma_{P_{i} \in \mathcal{P}} c_{i}=1, v^{\prime}(i)=k v(i)+c_{i}=0, \forall i \in \mathcal{P}
$$

has determinant $v(\mathcal{P})-\Sigma_{P_{i} \in \mathcal{P}} c_{i}$. In this case $k=1 /\left(v(\mathcal{P})-\Sigma_{P_{i} \in \mathcal{P}} c_{i}\right)$ and $c_{i}=-k v(i)$.

The relation $\approx$ is an equivalence relation among the essential cooperative games with the same set of players $\mathcal{P}$. Then, for any equivalence class $K$ with respect to $\approx$, we have a unique cooperative game $C=(\mathcal{P}, v) \in K$, called normal element of $K$, such that $v(i)=0, \forall i \in \mathcal{P}$ and $v(\mathcal{P})=1$.

\section{Relations between cooperative games and geometric spaces}

Let $C=(\mathcal{P}, v)$ be an essential cooperative game in normal form. In [38], chap. $\mathrm{X}$, the authors introduce the simple games.

Definition 4.1. A cooperative game $C=(\mathcal{P}, v)$ is a simple game if one can partition $\mathcal{P}$ in two non-empty subsets: $\mathcal{W}$, the winning coalitions, and $\mathcal{L}$, the losing coalitions such that

- the complement of a winning coalition is a losing coalition and vice versa;

- a superset of a winning coalition is a winning coalition.

In terms of characteristic function, $C=(\mathcal{P}, v)$ is a simple game, in normal form, if $v(\mathcal{P})=1$ and, for every coalition $A$,

$$
v(A) \in\{0,1\}, v(A)=0 \Leftrightarrow v(-A)=1 .
$$

An example of simple game is given by the Fano plane, where the winning coalitions are the lines and their supersets. 
An extension of the simple games is considered in [31], [33]. In these papers the semi-simple games are considered where the set of coalitions is partitioned in three subsets: $\mathcal{W}$, the winning coalitions, $\mathcal{L}$, the losing coalitions, and $\mathcal{B}$, the blocking coalitions. Shapley, in [33], introduces a definition of semi-simple game.

Definition 4.2. A pair $C=(\mathcal{P}, \mathcal{W})$ is a semi-simple cooperative game with $\mathcal{P}$ the set of players and $\mathcal{W}$ the set of winning coalitions if the following axioms hold:

(W1) $\mathcal{P} \in \mathcal{W}$;

(W2) $\forall A, B \in \mathcal{P},(A \in \mathcal{W}, A \subseteq B) \Rightarrow B \in \mathcal{W}$;

(W3) $\forall A \in \mathcal{P}, A \in \mathcal{W} \Rightarrow-A \notin \mathcal{W}$.

The complement - $A$ of a winning coalition $A$ is said to be a losing coalition. A coalition $B$ is said to be a blocking coalitions if it is not a winning or a losing coalition.

In terms of characteristic function in normal form, a semi-simple cooperative game is a game $C=(\mathcal{P}, v)$ where $v(A)=1$ if $A$ is a winning coalition and $v(A)=0$ otherwise. Then, for every coalition $A$, we can have three possibilities. $A$ is said to be:

winning if $v(A)=1, v(-A)=0$;

losing if $v(A)=0, v(-A)=1$;

blocking if $v(A)=0, v(-A)=0$.

Now we examine some general relations between cooperative semi-simple games and geometries of rank 2. Firstly, let us introduce some definitions.

Definition 4.3. Let $\mathcal{P}$ be a non-empty set, whose elements are called points and let $\mathcal{F}$ be a family of non-empty subsets of $\mathcal{P}$. We say that:

IP $\mathcal{F}$ has the intersection property if $\forall A, B \in \mathcal{F}, A \cap B \neq \emptyset$;

NI $\mathcal{F}$ has the non-inclusion property if $\forall A, B \in \mathcal{F},(A \subseteq B) \vee(B \subseteq A) \Rightarrow A=$ $B$;

CL the closure of $\mathcal{F}$ is the family $K(\mathcal{F})$ of the subsets of $\mathcal{P}$ containing at least one element of $\mathcal{F}$. In this case $\mathcal{F}$ is said to be a generator of $K(\mathcal{F})$.

We prove the following theorem. 
Theorem 4.1. Let $\mathcal{P}$ be a non-empty set, whose elements are called players and let $\mathcal{F}$ be a family of non-empty subsets of $\mathcal{P}$. If $\mathcal{F}$ has the intersection property then its closure $\mathcal{W}$ satisfies the axioms of Shapley for the winning coalitions. Moreover, if $\mathcal{F}$ is a minimal generator of $\mathcal{W}$ then the non-inclusion property holds.

Proof Let $\mathcal{W}$ be the closure of $\mathcal{F}$. Then the properties (W1) and (W2) are evident. If $A \in \mathcal{W}$ then $\exists B \in \mathcal{F}: B \subseteq A$. For the intersection property, $\forall C \in \mathcal{F}, C \cap A \supseteq C \cap B \neq \emptyset$ and then $-A$ not contains $C$. It follows that $-A \notin \mathcal{W}$. Moreover, if the non-inclusion property is not valid, there exists $A, B \in \mathcal{F}$ such that $A \subset B$. In this case $\mathcal{F}-\{B\}$ is a generator of $\mathcal{W}$ and then $\mathcal{F}$ is not a minimal generator.

Let $G=(\mathcal{P}, \mathcal{B}, I)$ be a projective plane. The set of lines $\mathcal{B}$ satisfies the intersection and non-inclusion properties. Then we have the following corollary.

Corollary 4.1. Let $G=(\mathcal{P}, \mathcal{B}, I)$ a projective plane of order $q$. The family $\mathcal{W}$ of the sets $S \subseteq \mathcal{P}$ containing at least a line (i.e. all the points incident with a line) satisfies the properties of Shapley for the set of winning coalition.

Theorem 4.2. Let $G=(\mathcal{P}, \mathcal{B}, I)$ be a geometry of rank 2. The family $B$ has the non-inclusion property. The family $\mathcal{W}$ of the subsets of $\mathcal{P}$ containing at least a block and at least one point of every block satisfies the properties of Shapley for the set of winning coalition. The pair $C=(\mathcal{P}, \mathcal{W})$ is said to be the semi-simple cooperative game associated to $G$.

There are two types of possible blocking coalitions:

(T1) a blocking coalition of type (T1) is a subset of $\mathcal{P}$ not containing any block and having at least a point in common with each block;

(T2) a blocking coalition of type (T2) is a subset $\mathcal{P}$ containing at least one block and with empty intersection with at least one other block.

If the intersection property holds then there are not blocking coalitions of type (T2).

Proof The non-inclusion property is equivalent to the fact that every maximal flag has two elements. It is evident that the family $\mathcal{W}$ of the subsets of $\mathcal{P}$ containing at least a block and at least one point of every block satisfies the properties (W1) and (W2). Moreover, if $A$ is an element of $\mathcal{W}$, then contains at least a block $B$. Then its complement has no points of $B$ and so it is not in $\mathcal{W}$. If a subset $B$ of $\mathcal{P}$ is of type (T1) (resp. (T2)), then also $-B$ is of the same type and then $B$ is a blocking coalition. If the intersection property holds then a subset containing a block contains at least a point of every block 
and so is a winning coalition. In this case there are not blocking coalitions of type (T2)

Example 4.1 An economic/social interpretation. Let $G=(\mathcal{P}, \mathcal{B}, I)$ be a geometry of rank 2 . The elements of $\mathcal{P}$ are the players and the blocks are companies. A player incident to a company has a power of veto on the decisions taken by the company, a coalition containing a block has the total control of the company represented by such block. If $C=(\mathcal{P}, \mathcal{W})$ is the cooperative game associated to $G$, then a winning coalition is a subset of $\mathcal{P}$ containing a company and having a power of veto for every company. A blocking coalition of type (T1) is a coalition not containing any company but having at least a player in common with each company; a blocking coalition of type (T2) is a coalition having a total control of at least a company but without power of veto in at least a company.

Example $4.2 \mathrm{~A}$ dual economic/social interpretation. Let $G=(\mathcal{P}, \mathcal{B}, I)$ be a geometry of rank 2 . The elements of $\mathcal{P}$ are the individuals and the blocks, i.e. the companies, are the players. A coalition $K$ of companies is winning if contains all the companies incident with an individual and for every individual $P \in \mathcal{P}$ there exists a company belonging to $K$ and incident with $P$. A blocking coalition of type (T1) is a set $H$ of companies such that for every individual $P$ there exist a $B \in H$ and a $C \in-H$ incident to $P$. A blocking coalition of type (T2) is a coalition having a total control of at least an individual but not incident with at least an individual.

From a geometric point of view, if $G=(\mathcal{P}, \mathcal{B}, I)$ is a geometry of rank 2 , then the blocking coalitions of type (T1) are called blocking sets. Many authors have dealt with the search of blocking sets, especially in projective planes, (see, e.g., [2], [3], [4], [5], [6], [8], [9], [10], [11], [12], [22], [23], [24], [35], $[37])$.

Definition 4.4. Let $C=(\mathcal{P}, \mathcal{W})$ be a semi-simple game and let $\mathcal{B}$ be the set of minimal winner coalitions. The geometry, of rank $2, G=(\mathcal{P}, \mathcal{B}, I)$, where, $\forall x \in \mathcal{P}, y \in \mathcal{B}, x I y \Leftrightarrow x \in y$ is said to be the geometry associated to $C$.

Corollary 4.2. Let $C=(\mathcal{P}, \mathcal{W})$ be a semi-simple game and let $G=(\mathcal{P}, \mathcal{B}, I)$ be the associate geometry. Then $\mathcal{B}$ has the intersection and non-inclusion properties.

\section{Winning and blocking coalitions at level $\alpha$}

Let $C=(\mathcal{P}, v)$ be an essential cooperative game in normal form. For every $\alpha \in[0,1]$ such that $\alpha>0.5$, let $\beta=1-\alpha$. From (C2), for every coalition $A \in 2^{\mathcal{P}}$, we have the following possibilities: 
(L1) $v(A) \geq \alpha$ and $v(-A) \leq \beta$;

(L2) $v(A) \leq \beta$ and $v(-A) \geq \alpha$;

(L3) $v(A) \leq \beta$ and $v(-A) \leq \beta$;

(L4) $v(A) \leq \beta$ and $\beta<v(-A)<\alpha$;

(L5) $\beta<v(A)<\alpha$ and $v(-A) \leq \beta$.

Let us introduce the following definition:

Definition 5.1. The coalition $A$ is said to be a winning coalition at level $\alpha$ if (L1) holds and a losing coalition at level $\alpha$ if (L2) holds. The set $A$ is a blocking coalition al level $\alpha$ in the other cases. A blocking coalition is a strict blocking coalition if (L3) holds.

By previous definitions, for every $\alpha>0.5$ we have:

Theorem 5.1. The set $\mathcal{W}_{\alpha}$ of winning coalitions at level $\alpha$ satisfies the Shapley properties (W1), (W2) and (W3), then $C_{\alpha}=\left(\mathcal{P}, \mathcal{W}_{\alpha}\right)$ is a semi-simple cooperative game. Let us call it $\alpha$-game generated by $C=(\mathcal{P}, v)$.

Proof From (W1) and (L1), the big coalition $\mathcal{P}$ is a winning coalition at level $\alpha$ and the empty coalition $\emptyset$ is a losing coalition. Moreover:

- $A$ is a winning coalition at level $\alpha$ if and only if his complement $-A$ is a losing coalition;

- If $A$ is a winning coalition at level $\alpha$ and $B \supseteq A$ then also $B$ is a winning coalition at level $\alpha$.

Proposition 5.1. A is a blocking (resp. strict blocking) coalition at level $\alpha$ if and only if his complement $-A$ is a blocking (resp. strict blocking) coalition at level $\alpha$.

Definition 5.2. Let $C=(\mathcal{P}, v)$ be an essential cooperative game in normal form. For every $\alpha>1 / 2$, the $\alpha$-geometry generated by $C$ is the geometry $G_{\alpha}=\left(\mathcal{P}, \mathcal{B}_{\alpha}, I_{\alpha}\right)$ associated to the $\alpha$-game $C_{\alpha}=\left(\mathcal{P}, \mathcal{W}_{\alpha}\right)$. 


\section{Blocking coalitions in Steiner systems}

Definition 6.1. A finite geometry, of rank $2, G=(\mathcal{P}, \mathcal{B}, I)$ is said to be a design of type $t-(v, k, \lambda), 2 \leq t \leq k<v$, we write also $G \sim S\left(t_{\lambda}, k, v\right)$, if:

(D1) $\mathcal{P}$ has v elements;

(D2) (equicardinality) every block has $k$ points;

(D3) (balancing) through $t$ distinct points pass $\lambda$ blocks.

The following theorem applies (see, e.g. [16]):

Theorem 6.1. Let $b$ the number of blocks and $b_{i}$ the number of blocks through $i, i \leq t$ distinct points. We have the following relations:

- $v(v-1) \ldots(v-t+1) \lambda=b k(k-1) \ldots(k-i+1)$;

- $(v-i) \ldots(v-t+1) \lambda=b_{i}(k-i) \ldots(k-t+1)$, for $1 \leq i<t$;

- $b_{t}=\lambda, v b_{1}=k b, 1<i<t \Rightarrow(v-i) b_{i+1}=(k-i) b_{i}$.

In particular, for $\lambda=1$ a design $G \sim S\left(t_{1}, k, v\right)$ is said to be Steiner system $G \sim S(t, k, v)$. For $\lambda=1, t=2$ we have a Steiner system of lines, and the blocks are called lines.

Corollary 6.1. Let $G \sim S(t, k, v), b$ the number of blocks and $b_{i}$ the number of blocks through $i, i \leq t$, distinct points. We have the following relations:

- $v(v-1) \ldots(v-t+1)=b k(k-1) \ldots(k-i+1)$;

- $(v-i) \ldots(v-t+1)=b_{i}(k-i) \ldots(k-t+1)$, for $1 \leq i<t$;

- $b_{t}=1, v b_{1}=k b$.

Corollary 6.2. Let $G \sim S(2, k, v)$ a Steiner system of lines, $b$ the number of blocks and $b_{1}$ the number of blocks through a point. We have the following relations:

- $v(v-1)=b k(k-1)$;

- $(v-1)=b_{1}(k-1)$;

- $b_{2}=1$.

It is evident that in a Steiner system of lines $G \sim S(2, k, v), k \leq b_{1}$. If $k=b_{1}$, then the Steiner system reduces to a projective plane, and if $k=b_{1}-1$, $G$ is an affine plane. 
Definition 6.2. A blocking set on a Steiner system of lines $G \sim S(2, k, v)$ is a set of points not containing any line, but having at least a point in common with every line.

Definition 6.3. A winning coalition on a Steiner system of lines $G \sim S(2, k, v)$ is a set of points containing at least a line, and having at least a point in common with every line.

In particular we have the fundamental problems to find:

1. the minimal or maximal blocking sets;

2. the spectrum of the minimal blocking sets, that is the set of all the possible cardinalities of the minimal blocking sets;

3. the minimal winning coalitions;

4. the winning coalitions containing blocking coalitions.

By definitions it follows that the complement of a blocking set is also a blocking set, so to find the maximal blocking sets is equivalent to find the minimal ones.

Now we show some results in the particular case of projective planes.

It is well known that, in a non-degenerate finite projective plane, all the lines have the same number of points. If $q+1$ is such number, the projective plane is said to be of order $q$ and is noted $\pi_{q}$. Moreover, the lines through a fixed point $P$ are also $q+1$ and the points of $\pi_{q}$ are $q^{2}+q+1$.

It is well known that there exists a Desarguesian projective plane if and only if $q$ is a prime or a power of a prime and such plane is unique (see, e.g., [6], [19]). The first value of $q$ with non-Desarguesian planes is $q=9$.

For small values of $q$ we have:

- in $\pi_{2}$ there are not blocking sets;

- $\pi_{3}$ there are exactly two blocking sets;

- the blocking sets on $\pi_{4}$ and $\pi_{5}$ are classified, respectively, in papers of Berardi - Eugeni [2] and Berardi - Innamorati [5];

- the blocking sets on $\pi_{7}$ are classified in papers of Innamorati and Maturo (see [22], [23], [24]). If $k$ is the cardinality of a minimal blocking set on $\pi_{7}$ we have $12 \leq k \leq 19$. In particular there are, up to isomorphism, only two minimal blocking sets of order 12 and there is only a minimal blocking set with 19 points.

In the general case there are the following results ([22], [23], [24]), [29]): 
Theorem 6.2. Let $S(q)$ the spectrum of the minimal blocking sets in $\pi_{q}$. If $q \geq 4$, then $S(q) \supseteq[2 q-1,3 q-5] \cup\{3 q-3\}$ and, if $\pi_{q}$ is Desarguesian, $S(q) \supseteq[2 q-1,3 q-3]$.

Theorem 6.3. A sufficient condition for the existence of a minimal blocking set with $3 q-4$ points on a non-Desarguesian plane $\pi_{q}$ is that $\pi_{q}$ contains a proper subplane of order two.

In [28] H. Neumann conjectured that any finite non-Desarguesian plane contains a proper subplane of order two. By previous proposition, if the conjecture is true, we have that also for the non-Desarguesian plane of order $q$ there exists a blocking set with $3 q-4$ points.

\section{Conclusions, recommendations and perspective of re- search}

Two possible lines of research to deepen the relationship between geometric spaces and cooperative games are:

1. for every natural number $k \geq 2$ to define and study games associated with geometries of rank k;

2. to introduce games associated with algebraic hyperstructures.

Below we present some possible definitions and starting points for the development of these theories.

Let $G=\left(\Omega_{1}, \Omega_{2}, \ldots, \Omega_{k}, I\right)$ be a geometry of rank $k$. Let us introduce the following definition.

Definition 7.1. For every $i, j \in\{1,2, \ldots, k\}$, with $i \neq j$, a subset $W$ of $\Omega_{i}$ is said to be:

1. intersecting the elements of $\Omega_{j}$, if $\forall K \in \Omega_{j}, \exists x \in \Omega_{i}: x I K$;

2. covering an element of $\Omega_{j}$, if $\exists H \in \Omega_{j}:\left(x \in \Omega_{i}, x I H\right) \Rightarrow x \in W$.

A subset $W$ of $\Omega_{i}$ is said to be:

1. a winning coalition of type $i$ with respect to $\Omega_{j}$, if it intersects the elements of $\Omega_{j}$ and covers an element of $\Omega_{j}$;

2. a losing coalition of type $i$ with respect to $\Omega_{j}$, if it is the complement of a winning coalition;

3. a blocking coalition of type (T1) or blocking set, if it intersects the elements of $\Omega_{j}$, but not covers any element of $\Omega_{j}$; 
4. a blocking coalition of type (T2), if it covers an element of $\Omega_{j}$, but not intersects the elements of $\Omega_{j}$.

If we consider, $\forall i, j, i \neq j$ the winning coalitions of type $i$ with respect to $\Omega_{j}$, we obtain $n(n-1)$ semi-simple cooperative games. The study of relationships between such games can be an important line of research.

Generalizing the above definitions we can also define winning, losing and blocking coalitions of type $i$ with respect to a set $\left\{\Omega_{j_{1}}, \Omega_{j_{2}}, \ldots, \Omega_{j_{r}}\right\}$ not containing $\Omega_{i}$. If $r>1$ we can define two types of winning coalitions.

Definition 7.2. A subset $W$ of $\Omega_{i}$ is said to be:

1. a weak winning coalition of type $i$ respect to $\left\{\Omega_{j_{1}}, \Omega_{j_{2}}, \ldots, \Omega_{j_{r}}\right\}$, if it is intersecting the elements of all the $\Omega_{j_{s}} \in\left\{\Omega_{j_{1}}, \Omega_{j_{2}}, \ldots, \Omega_{j_{r}}\right\}$ and covering an element of at least an $\Omega_{j_{s}}$;

2. a strong winning coalition of type $i$ respect to $\left\{\Omega_{j_{1}}, \Omega_{j_{2}}, \ldots, \Omega_{j_{r}}\right\}$, if it is intersecting the elements of all the $\Omega_{j_{s}} \in\left\{\Omega_{j_{1}}, \Omega_{j_{2}}, \ldots, \Omega_{j_{r}}\right\}$ and covering an element of every $\Omega_{j_{s}}$;

A second line of research can be obtained by considering geometries associated to algebraic hyperstructures. For the concepts and fundamental theorems on algebraic hyperstructures, see e. g. [17], [15], [18], [27].

Let $(S, \circ)$ an algebraic hyperstructure, i.e. a nonvoid set $S$ with a function o that to every pair $(x, y)$ of elements of $S$ associates a nonvoid subset of $S$, denoted with $x \circ y$. Let us call "points" the elements of $S$ and "blocks" the hyperproducts $x \circ y$. If we introduce a suitable incidence relation $I$ we can have a rank 2 geometry $G=(S, \mathcal{B}, I)$, where $\mathcal{B}$ is the set of the blocks.

An incidence relations is the "relation of belonging" defined as follows: $\forall x \in S, x I x$ and $\forall x \in S, H \in \mathcal{B},(x I H \Leftrightarrow x \in H)$.

A different incidence relation is defined as follows: $\forall x \in S, x I x$ and $\forall x \in$ $S, H \in \mathcal{B},(x I H \Leftrightarrow(\exists y \in S: x \circ y=H))$.

We obtain two distinct rank 2 geometries and we can study the associated cooperative games and the relations between the winnings coalitions of such games.

Another line of research is obtained by considering fuzzy geometries, in which the degree of incidence between two elements is a number belonging to the interval $[0,1]$, and defining associated cooperative games. Some topics that we can put in connection with such research are the $\alpha$-geometries defined in Sec. 5 and the fuzzy algebraic hyperstructures $(S, \circ)$ in which $x \circ y$ is a nonvoid fuzzy subset of $S$. 


\section{Acknowledgements}

The publication of this paper is supported by the grant of CNCS (Romanian National Council of Research): PN-II-ID-WE - 2012 - 4 -161.

\section{References}

[1] K. J. Arrow, Social Choose and Individual Values, J. Wiley and Sons, New York, 1951, 2nd ed 1963.

[2] L. Berardi, F. Eugeni, Blocking sets in projective plane of order four, Ann. of Discrete Math. 37 (1988), 43-50.

[3] L. Berardi, F. Eugeni, Blocking sets e Teoria dei Giochi, Atti Sem. Mat. Fis. Univ. Modena 34 (1988), 165-196.

[4] L. Berardi, F. Eugeni, Blocking sets and game theory II, Proc. "Blocking sets", Gissen, 1989, Springer.

[5] L. Berardi, S. Innamorati, Irreducible blocking sets in the projective plane of order five, Atti Sem. Mat. Fis. Univ. Modena, 38 (1990), 293-311.

[6] A. Beutelspacher, Blocking sets and partial spreads in finite projective spaces, Geom. Dedicata 9 (1980), 425-449.

[7] A. Beutelspacher, U. Rosenbaum, Projective Geometry, Cambridge University Press, 1998

[8] A. Blokhuis, Blocking sets in Desarguesian planes, Proc. Int. Conf. Comb. "Paul Erdos is Eighty", Bolyai Soc. Math. Studies, (1993), 1-20.

[9] A. Blokhuis, A. E. Brower, Blocking sets in Desarguesian projective planes, Bull. London Math. Soc. 18 (1986), 132-134.

[10] A. A. Bruen, Baer subplanes and blocking sets, Bull. Amer. Math. Soc. 76 (1970) 342-344.

[11] A. A. Bruen, Blocking sets in projective planes, SIAM J. Appl. Math. 21 (1971), 380-392.

[12] A. A. Bruen, J. A. Thas, Blocking sets, Geom. Dedicata 6 (1977), 193-203.

[13] E. Burger, Introduzione alla teoria dei giochi, Franco Angeli editore, 1967

[14] P.J.Cameron, Four lectures on finite geometry, in Finite geometries, editors C. A. Baker, L.M.Batten, Lecture Notes in Pure and Appl. Math. 103, M.Dekker, New York, 1985, 27-63. 
[15] J. Chvalina, S. Hoskova-Mayerova and A. D. Nezhad, General actions of hyperstructures and some applications, Analele Univ. Ovidius Constanta, vol. 21 (1) 2013, pp.59 - 82

[16] M. Cerasoli, F. Eugeni, M. Protasi, Introduzione alla Matematica Discreta, Zanichelli, Bologna, 1988

[17] P. Corsini, V. Leoreanu, (2003), Applications of Hyperstructure Theory. Kluwer AP, Dordrecht, Boston.

[18] I. Cristea, S. Jancic-Rasovic, Composition hyperrings Analele Univ. Ovidius Constanta, vol. 21 (2) 2013, pp.81 - 94.

[19] J. W. P. Hirschfeld, Projective geometries over finite fields, Clarendon Press, Oxford, 1979.

[20] A. J. Hoffman, M. Richardson, Block design games, Canad. J. Math. 13 (1961), 110-128

[21] S. Innamorati, Sulle coalizioni in teoria dei giochi, Ratio Math. 2 (1991), $139-150$

[22] S. Innamorati, A. Maturo, On irreducible blocking sets in projective planes, Ratio Math. 2, (1991), 151-155.

[23] S. Innamorati, A. Maturo, On blocking sets of smallest cardinality in the projective plane of order seven, Combinatorics '88, Mediterranean Press, Cosenza, Italy, 1991, 79-96.

[24] S. Innamorati, A. Maturo, The spectrum of minimal blocking sets, Discrete Mathematics, 208/209 (1999) 339-347

[25] S. Innamorati, F. Zuanni, Minimum blocking configurations, J. Geom. 55 (1996) 86-98.

[26] R. D. Luce, H. Raiffa, Games and Decisions, John Wiley, New York, 1957.

[27] A. Maturo, Cooperative Games, Finite Geometries and Hyperstructures, Ratio Mathematica, 14, 2003, 57-70.

[28] H. Neumann, On some finite non-Desarguesian planes, Arch. Math., 6 (1955), 36-40.

[29] T. G. Ostrom, F. A. Sherk, Finite projective planes with affine subplanes, Canad. Math. Bull. 7 (1964), 549-560.

[30] G. Oven, Game Theory, Academic Press, New York, 1982 
[31] M. Richardson, On finite projective games, Proc. Amer. Math. Soc., 7 (1956), 458-465

[32] J. Rosenmuller, Some topics of cooperative game theory, in Modern Applied Math., North Holland, 1982

[33] L. S. Shapley, Simple Games - An outline of the theory, Rand Corporation P-series Report, 1962

[34] J. Singer, A theorem in finite geometry and some application to number theory, Trans. Amer. Math. Soc. 43 (1938), 377-385.

[35] T. Szonyi, Note on existence of large minimal blocking sets in Galois planes, Combinatorica 12 (1992), 227-235.

[36] G. Tallini, Geometrie di incidenza e matroidi, IAC Roma, Quad. Serie III-N.127 (1981), 1-34.

[37] G. Tallini, On blocking sets in finite projective and affine spaces, Ann. of Discrete Math. 37 (1988), 433-450.

[38] J. Von Neumann, O. Morgenstern, Theory of games and economic behaviour, Princeton University Press, Princeton, 1947.

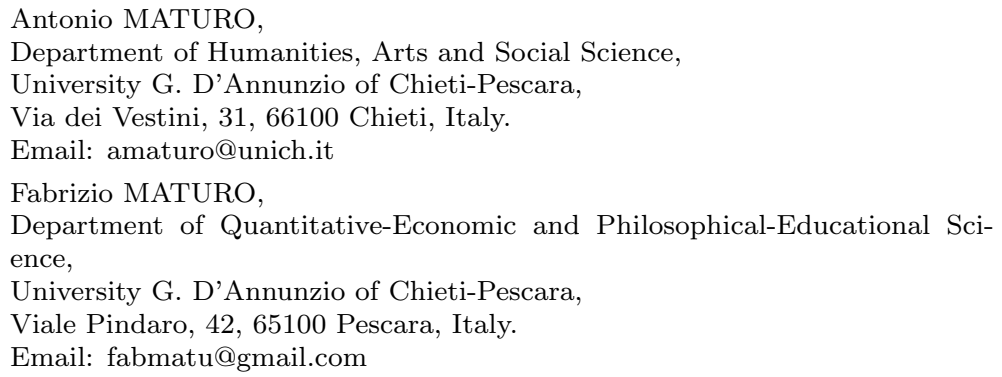

